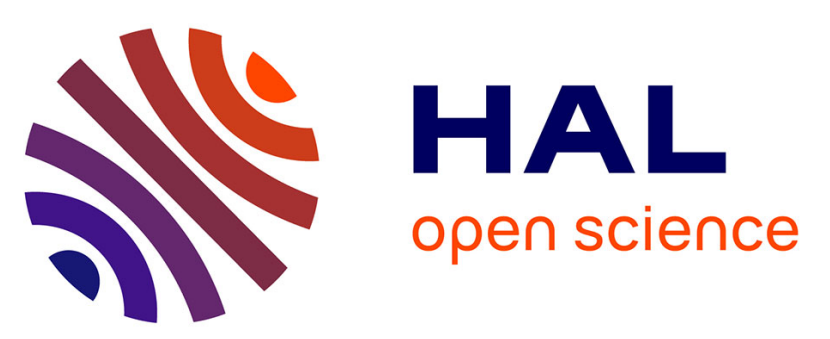

\title{
FREE-FORM ANISOTROPY: A NEW METHOD FOR CRACK DETECTION ON PAVEMENT SURFACE IMAGES
}

Tien Sy Nguyen, Stéphane Begot, Florent Duculty, Manuel Avila

\section{- To cite this version:}

Tien Sy Nguyen, Stéphane Begot, Florent Duculty, Manuel Avila. FREE-FORM ANISOTROPY: A NEW METHOD FOR CRACK DETECTION ON PAVEMENT SURFACE IMAGES. 18th IEEE International Conference on Image Processing, Sep 2011, Bruxelles, Belgium. hal-00626896

\section{HAL Id: hal-00626896 https://hal.science/hal-00626896}

Submitted on 27 Sep 2011

HAL is a multi-disciplinary open access archive for the deposit and dissemination of scientific research documents, whether they are published or not. The documents may come from teaching and research institutions in France or abroad, or from public or private research centers.
L'archive ouverte pluridisciplinaire HAL, est destinée au dépôt et à la diffusion de documents scientifiques de niveau recherche, publiés ou non, émanant des établissements d'enseignement et de recherche français ou étrangers, des laboratoires publics ou privés. 


\title{
FREE-FORM ANISOTROPY: A NEW METHOD FOR CRACK DETECTION ON PAVEMENT SURFACE IMAGES
}

\author{
Tien Sy NGUYEN ${ }^{(1,2)}$, Stéphane BEGOT ${ }^{(1)}$, Florent DUCULTY ${ }^{(1)}$, Manuel AVILA ${ }^{(1)}$
}

\section{(1) PRISME Laboratory, University of Orleans, France, (2) Vectra road engineering, www.vectra.fr Email: manuel.avila@univ-orleans.fr}

\begin{abstract}
This paper presents a new measure which takes into accounts simultaneously brightness and connectivity, in the segmentation step, for crack detection on road pavement images. Features which are calculated along every free-form paths provide detection of cracks with any form and any orientation. The method proposed does not need learning stage of free defect texture to perform default detection. Experimental results were conducted on some samples of different kinds of pavements. Results of the method are also given on other kinds of images and can provide perspectives on other domains as road extraction on satellite images or segment blood vessels in retinal images.
\end{abstract}

Index Terms - Image segmentation, crack detection, defect detection, texture analyses

\section{INTRODUCTION}

From 1990, there is a growing interest in pavement defect detection using image processing techniques [1]. Crack detection on pavement surfaces is a difficult problem due to the noisy pavement surfaces. There are different kinds of texture that can be encountered on road pavements. Cracks can have any form; crack size can be as small as 1 pixel in width and thinner than aggregate size. Figure 1 illustrates some road pavement image samples.

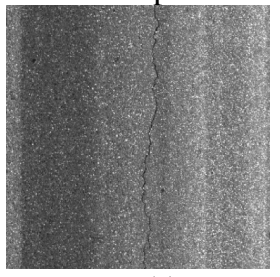

(a)

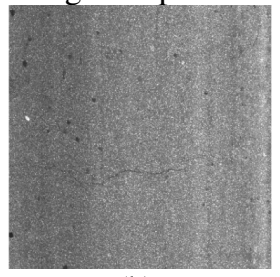

(b)

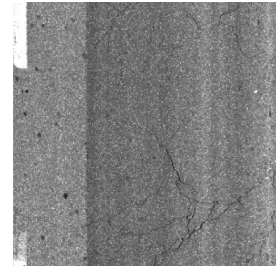

(c)
Figure 1: pavement image samples: (a) longitudinal crack, (b) transversal crack, (c) alligator crack.

The paper is organized as follows: In section 2, a short review of defect detection methods is proposed. In section 3, we introduce a new approach based on Free-Form Anisotropy (FFA) for segmentation. First, we recall Conditional Texture Anisotropy (CTA), which was introduced by F. Roli [2], and adapted for pavement crack detection [3]. Then, we explain FFA method which overcomes CTA limitations (orientations and linear form of crack). Section 4 summarizes experimental results. Finally, we conclude and propose others purposes for the method.

\section{REVIEW OF DEFECT DETECTION METHODS}

Because of the road pavement image nature, crack detection methods, in literature, were based on "stable" characteristics of cracks. We can give the two following characteristics of cracks [1], [4]:

- Brightness: crack pixels are darker than their neighbors.

- Form: crack is continuous or could be formed by various continuous segments. Its length is greater than its width and than granulate size.

Both of these characteristics can be noised with shadows, lane marking, etc.

Usually, crack pavement detection methods can be divided into four sequential stages: pre-processing, segmentation, post-processing and classification. According to [5], in most of existing methods, classification step is trivial due to the easy task consisting in separating different crack types (longitudinal, transversal and alligator).

Most of approaches, in literature, use brightness characteristic of crack for segmentation followed by a postprocessing step, which uses connectivity characteristic to connect crack segments and to eliminate noises.

Thresholding is frequently used to segment cracks, fixed threshold in [6-7] or fuzzy threshold in [8]. Some methods $[9,10]$ divide image into grid cells and then classify each cell as crack or crack-free cell by comparing mean and standard deviation of the cell with their neighbors or by UINTA filtering [11]. Authors in [5] supposed that, by applying a 2D Continuous Wavelet Transform (CWT), the differences between crack pixels and background pixels could be raised up.

After segmentation step, crack appears as discontinuous regions with noises. Post-processing step is needed to remove noise and to connect crack segments.

In case of use of grid cells, crack as thin as 1 pixel cannot be detected considering only statistical features of intensities. Use of wavelet [5] is a good approach by considering multi-resolution aspect, but their results showed that CWT not only rises up cracks but also noises. Recent approaches $[9,11]$ provide very noisy results for the 
segmentation step and it is hard to obtain good connection results.

In the next part, we propose a new method which takes into account simultaneously intensity and crack form features for segmentation step.

\section{FREE-FORM ANISOTROPY}

\subsection{Conditional Texture Anisotropy for crack detection}

Conditional Texture Anisotropy (CTA) was first introduced by F. Roli [2]. The main idea was to find out a measure which produces small values in one orientation (e.g. along crack orientation) and higher values in other orientations. Let $w_{1}$ be the class of defect-free pixels and $w_{2}$ be the class of default pixels. The CTA of a pixel $l$ can be defined as:

$$
\begin{aligned}
& C T A(l)=\frac{\max _{j}\left\{p\left(x_{j}^{l} \mid l \in w_{1}\right)\right\}-\min _{j}\left\{p\left(x_{j}^{l} \mid l \in w_{1}\right)\right\}}{\max _{j}\left\{p\left(x_{j}^{l} \mid l \in w_{1}\right)\right\}} \\
& \text { where : }
\end{aligned}
$$

$x_{j}^{l}=\left(\right.$ feature $_{1}^{l}$, feature $_{2}^{l}, \ldots$, feature $\left.{ }_{n}^{l}\right)$ is a set of $n$ texture features computed along the orientation $j$.

$p\left(x_{j}^{l} \mid l \in \mathrm{w}_{1}\right)$ is probability for the pixel $l$ to be a defect-free pixel along the orientation $\mathrm{j}$. In [2], $j$ is usually one of the 4 traditional orientations $\left(0^{\circ}, 45^{\circ}, 90^{\circ}, 135^{\circ}\right)$. According to crack characteristics, $p\left(x_{j}^{l} \mid l \in \mathrm{w}_{1}\right)$ should take low value on dominant orientation of crack. As well, $p\left(x_{j}^{l} \mid l \in \mathrm{w}_{1}\right)$ will take high values for other orientations. We can deduce from equation (3.1) that CTA takes high value (close to 1) on crack pixels and low value for defect-free pixels (close to 0).

According to "brightness" characteristic of crack and Gaussian-form histogram [5] of pavement image, mean and standard deviation of pixel intensity of oriented segment have been chosen as features to calculate CTA. These oriented segments are composed of $(2 d+1)$ pixels.

$$
h\left(\pi_{1}, \pi_{2}\right)=\sup \left\{\min \left(\pi_{1}, \pi_{2}\right)\right\}
$$
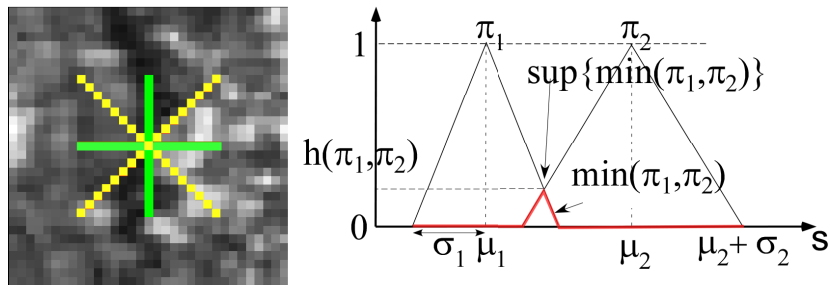

Figure 2 : oriented segments and sup-min function to evaluate degree of coherence between two sources.

To compute $p\left(x_{j}^{l} \mid l \in \mathrm{w}_{1}\right)$, we use possibility theory [13] to evaluate degree of coherence (3.2) between two sources (Figure 2). Source $\pi_{\mathrm{i}}$ is composed by mean $\mu_{\mathrm{i}}$ and standard deviation $\sigma_{i .}$.

We dispose of 4 sources, one for each orientation. To avoid training stage for background characterization, we use this hypothesis: crack affect texture only in one orientation (the crack orientation). Then to evaluate background (or defect-free) source, we compute mean of the 3 sources which have higher values (Figure 3).

A two levels threshold [3] is used to produce binary images. The most important parameter of the method is the distance $d$, of oriented segments, used to compute features.

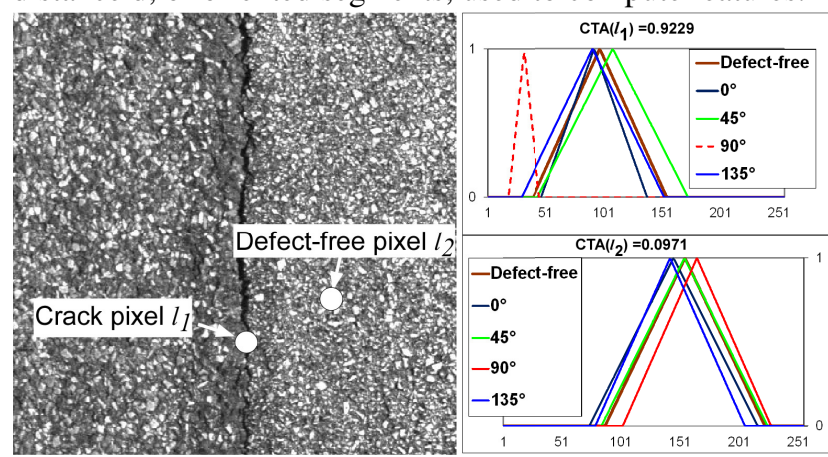

Figure 3: Computation of CTA for $l 1$ and $l 2$ using the degree of coherence (right figures).

In Figure 4, we see results of CTA, on an image, on which we produce synthetic defects. We create defects with 3 of the traditional orientations and one different. These defects go from 1 to 4 pixels in width, with intensities chosen randomly with values near their neighbors.

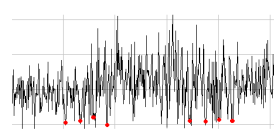

transversal profil

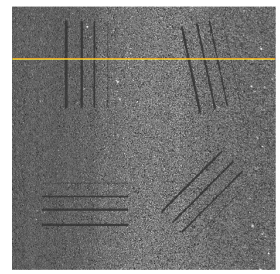

original image

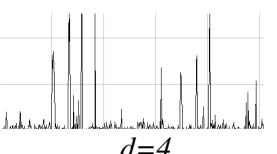

$d=4$

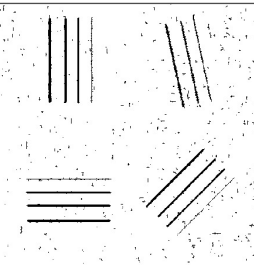

binary images
Figure 4: CTA results for different distances d.

In this Figure 4, we see that defects with traditional orientations are correctly detected. When distance $\mathrm{d}$ is high enough, the background noises disappear, but we see also that, for non traditional orientation, only large defects are detected.

So CTA is interesting for traditional orientations and it provides efficient background suppression when the distance $\mathrm{d}$ is high enough. But for other orientations, CTA suppresses also thin defects.

\subsection{Free-Form Anisotropy}

To overcome the CTA limitations, we propose the FreeForm Anisotropy method (FFA) which calculates, for each pixel, features along every free-form path.

\subsubsection{Definition}

We reach 4 minimal paths according to 4 global orientations as it is shown in figure 5. A minimal path is defined as a path for which sum of pixel intensities is the smallest. Graph theory, for example Dijkstra algorithm [14], provides 
solution to find efficiently these minimal paths.

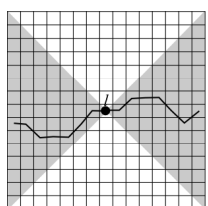

(a)

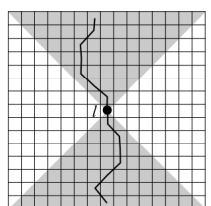

(b)

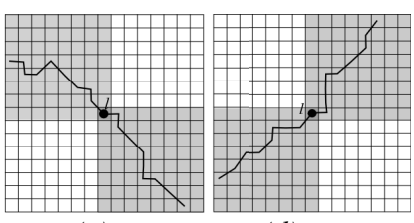

(c) (d)
Figure 5 : Minimal paths of pixel $l$ according to 4 orientations: (a) transversal, (b) longitudinal, (c) diagonal $135^{\circ}$, (d) diagonal $45^{\circ}$.

Features are calculated for each path and converted into sources and we can compute background source $\pi_{\mathrm{bgd}}$ as it was done for CTA.

Then the FFA of each pixel $l$ can be formulated as:

$$
F F A(l)=\frac{\max _{j}\left\{h\left(\pi_{j}, \pi_{b g d}\right)\right\}-\min _{j}\left\{h\left(\pi_{j}, \pi_{b g d}\right)\right\}}{\max _{j}\left\{h\left(\pi_{j}, \pi_{b g d}\right)\right\}}
$$

with $j$ a global orientation, and $h\left(\pi_{j}, \pi_{b g d}\right)$ computed on the 4 minimal paths (Figure 5) with pixel $l$ at the center of the path with $(2 d+1)$ length.

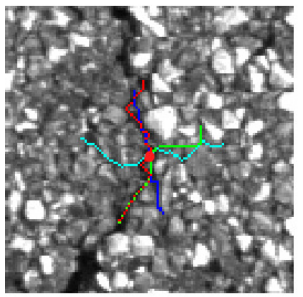

(a)

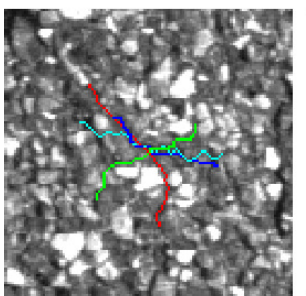

(c)

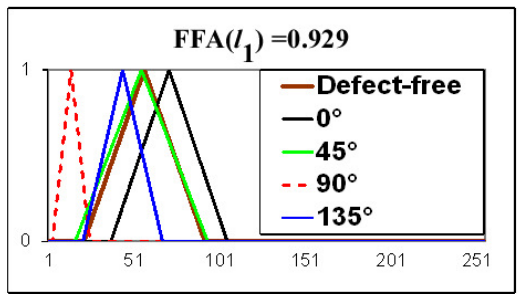

(b)

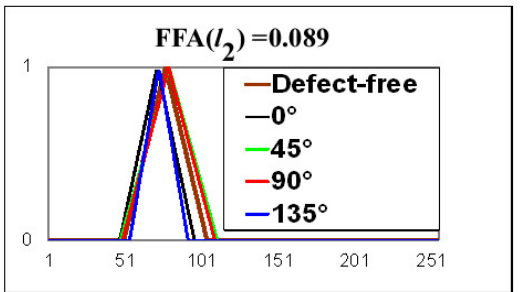

(d)
Figure 6 : Computation of FFA for 2 pixels width $d=30$. (a,b) crack pixel (c,d) defect-free pixel.

As for CTA measure, the FFA measure is close to 1 for crack pixels and close to 0 for defect-free pixels.

FFA computation is illustrated on figure 6 . We can see that the free-form path follows the crack. This shows the ability of FFA to explore with accuracy different crack forms. Without crack, minimal paths produce sources with high correlation level (Figure $6 \mathrm{~d}$ ).

The same strategy, as for CTA, was used to test FFA. Figure 7 shows the original image with synthetic cracks, FFA and binary images for different distances d, and profiles of line extracted on images. If distance $d$ is high enough, all cracks are detected in any orientation and with the minimal width (1 pixel).

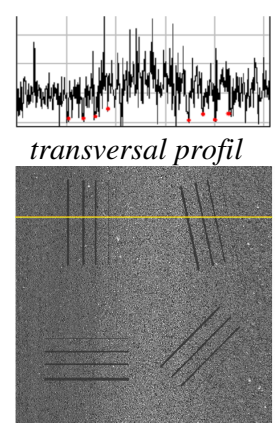

original image

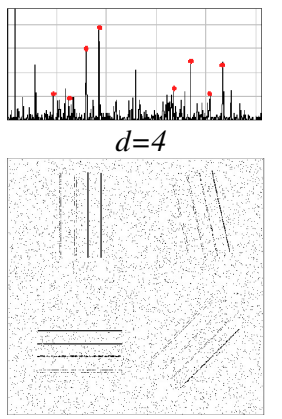

binary images
Figure 7 : FFA results for different distances $d$.

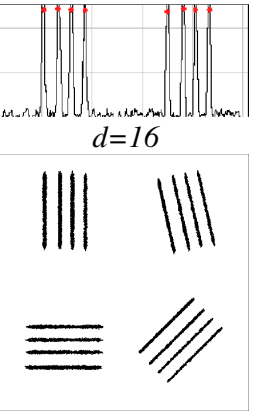

\section{RESULTS}

\subsection{Comparative results}

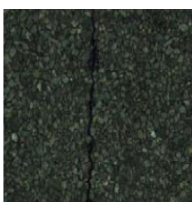

(a)

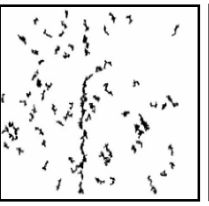

(b)

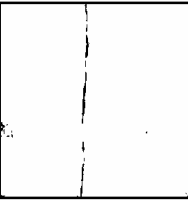

(c)

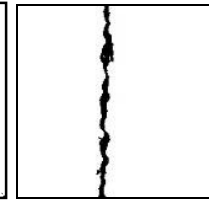

(d)
Figure 8 : Anisotropy vs. Subirat's 2D CWT. Inspected image (a), Subirat's CWT (b), CTA (c), FFA (d).

Figure 8 (a) shows a longitudinal crack on real image of road pavement. In this example, we compare CTA and FFA with 2D CWT method [9]. Both CTA results and FFA results (Figure $8 \mathrm{c}$ and $\mathrm{d}$ ) contain less of noise. This is the ability of the segmentation step to take into account intensity, form and connectivity of the default. FFA is better than CTA for connecting crack segments.

\subsection{Texture variation}

We use different kinds of pavement images with different properties. Table 1 gives some of theses textures attributes for each kind of texture. We see granulate size, contrast. The two last parameters are Haralick attributes extracted from co-occurrence matrix [15].

\begin{tabular}{|c|c|c|c|c|}
\hline Image & $\begin{array}{c}\text { Granulate size } \\
(\mathrm{mm}) \mathrm{min} / \mathrm{max}\end{array}$ & Contrast & Correlation & Entropy \\
\hline 1 & $0 / 4$ & 308.1 & 0,001 & 8,7 \\
\hline 2 & $0 / 10$ & 537,3 & 0,0005 & 9,38 \\
\hline 3 & $0 / 13$ & 612,4 & 0,0032 & 9,4 \\
\hline 4 & $0 / 18$ & 876,72 & 0,00031 & 9,9 \\
\hline
\end{tabular}

Table $1:$ Texture attributes of selected images.

On these images (Figure 9), we generate synthetic cracks with the same method as in 3.1. Cracks have "no segment" forms and intensities are chosen randomly.

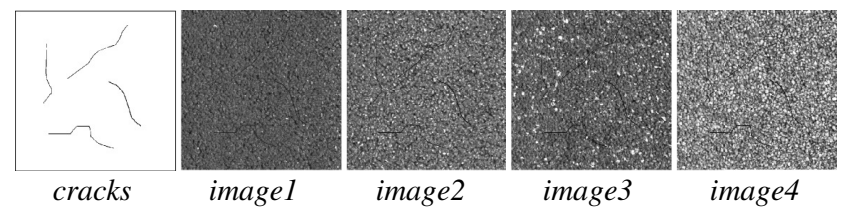

Figure 9 : Synthetic cracks on different kinds of pavement images. 
In Figure 10, we show FFA results for different distances d. In each case, all cracks are fully detected. If distance is high enough, there is no noise detection. The method is able to perform background suppression for different kinds of textures. This demonstrates the robustness of the method.

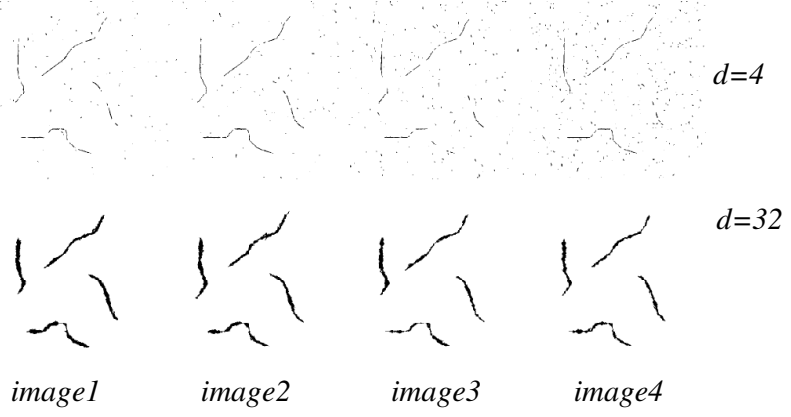

Figure 10: FFA results on different kinds of pavement images.

\subsection{Results on real defects on pavement images}

These tests were performed on real 16473 images (Figure 11) captured dynamically [3-4]. We obtained $93.6 \%$ detection rate and $13.7 \%$ false alarm with FFA. With CTA method, we obtained only $73.8 \%$ detection rate and more than $27.6 \%$ false alarm. Computation time for these high resolution images (2048x2048 pixels) is about 20 seconds (Dell Precision PWS670, Xeon 3.6 GHz, RAM 4Go).
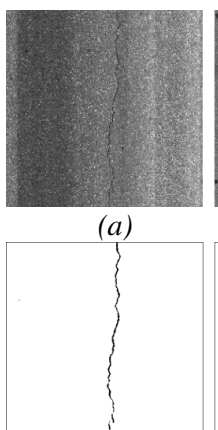

(a)

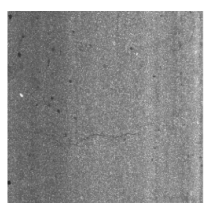

(b)

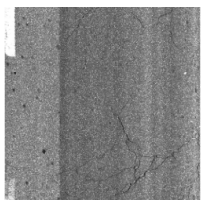

(c)

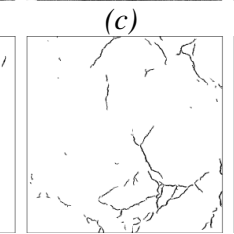

(c)

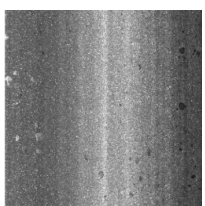

(d)
Figure 11 : FFA results. (a) Longitudinal crack, (b) transversal crack, (c) alligator crack, (d) defect-free.

In this figure, all defects (figure $11 \mathrm{a}, \mathrm{b}, \mathrm{c}$ ) are detected. We see details of alligator crack (c). Without defect (figure $11 \mathrm{~d}$ ), nothing is detected. and we see the efficiency of the method which provides results with very low noise.

\section{CONCLUSION AND PERSPECTIVES}

In this paper, we have introduced a new method for crack detection on road pavement images. By considering all characteristics of crack and by unrestricting crack orientations and forms, the method provides good results on crack segmentation. Cracks which are as small as $1 \mathrm{~mm}(1$ pixel in width) could be detected with any form and orientations.

Fine structures on other kinds of images like medical images (Figure 12 a) or satellite images (Figure 12 c) have similar characteristics as pavement cracks. Results show that FFA can also be useful on these kinds of images with fine structures.

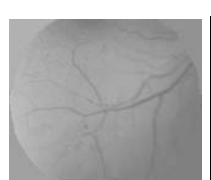

(a)

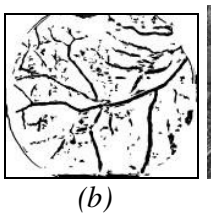

(b)

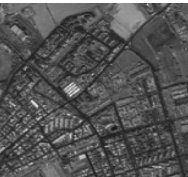

(c)

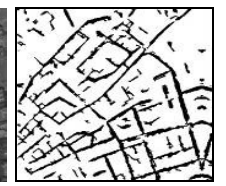

(d)
Figure 12 : FFA applied on other kind of fine structure extraction, blood vessels in retinal images $(a, b)$, road extraction on satellite images (c, d).

Characteristics of these fine structures in other random texture surfaces are similar to cracks on pavement surfaces. Good results obtained on some images of this kind suggest good perspectives for using FFA in other domains as: ceramic damages detection, road network extraction in satellite images and blood vessels segmentation in retinal images.

\section{REFERENCES}

[1] Schmidt B., Automated Pavement Cracking Assessment Equipment State of the Art. 2003, World Road Association (PIARC).

[2] Roli F., Measure of texture anisotropy for crack detection on textured surfaces, in Electronics Letters. 1996. pp. 1274-1275.

[3] Nguyen T.S., M. Avila, and S. Begot, Automatic defect detection on road pavement using anisotropy measure, in Proceedings EUSIPCO. 2009.

[4] Nguyen T.S., et al., Detection of defects in road surface by a vision system, in Proc. 14th IEEE Mediterranean Electrotechnical Conference MELECON 2008. 2008. pp. 847-851.

[5] Chambon S., J. Dumoulin, and P. Subirats, Introduction of a wavelet transform based on 2D matched filter in a Markov Random Field for fine structure extraction: Application on road crack detection, in SPIE Conference on Image Processing: Machine Vision Applications II, San Jose, United-States. 2009.

[6] Nallamothu S. and K.C.P. Wang, Experimenting with Recognition Accelerator for Pavement Distress Identification, in Transportation Research Record. 1996. pp. 130-135.

[7] Chua K.M. and L. Xu, Simple Procedure for Identifying Pavement Distresses from Video Images, in J. Transp. Engrg. 1994. pp. 412-431.

[8] Cheng, .D., et al., Novel Approach to Pavement Cracking Detection Based on Fuzzy Set Theory, in Journal of Computing in Civil Engineering. 1999. pp. 270-280.

[9] Oliveira H., Correia P.L., Identifying and retrieving distress images from road pavement surveys, in Proc. 15th IEEE International Conference on Image Processing ICIP 2008. pp. 57-60.

[10]Huang Y. and B. Xu, Automatic inspection of pavement cracking distress, in Journal of Electronic Imaging. 2006.

[11]Oliveira H.; JJC Caeiro; Correia, P.L.; Improved Road Crack Detection Based on One-class Parzen Density Estimation and Entropy Reduction, Proc IEEE International Conf. on Image Processing ICIP 2010, pp 2201-2204.

[12]Howe, R. and G.C.a. Gerardo, An assessment of the feasibility of developing and implementing an automated pavement distress survey system incorporating digital image processing. 1997, The Virginia Transportation Research Council.

[13]Dubois D. and H. Prade, On the use of aggregation operations in information fusion processes. Fuzzy Sets and Systems. 2004. 142,143161.

[14]Thomas, H.C., et al., Introduction to Algorithms. 2001: McGraw-Hill Higher Education.

[15]Theodoridis S. and K. Koutroumbas, Pattern Recognition, Fourth Edition. 2008: Academic Press. pp 418. 